\title{
Outcomes of living donor kidney transplantation in diabetic patients: age and sex matched compar- ison with non-diabetic patients
}

\author{
Chung Hee Baek ${ }^{1}$, Hyosang Kim¹ ${ }^{1}$, Seung Don Baek ${ }^{1}$ Mun Jang ${ }^{1}$, Wonhak Kim ${ }^{1}$ Won Seok Yang , \\ Duck Jong Han ${ }^{2}$, and Su-Kil Park ${ }^{1}$
}

${ }^{1}$ Division of Nephrology, Department of Internal Medicine, ${ }^{2}$ Department of Surgery, Asan Medical Center, University of Ulsan College of

Medicine, Seoul, Korea
Received: March 10, 2016

Revised : May 24, 2016

Accepted: May 30, 2016

\section{Correspondence to}

Su-Kil Park, M.D.

Division of Nephrology,

Department of Internal

Medicine, Asan Medical Center, University of Ulsan College of

Medicine, 88 Olympic-ro 43-gil,

Songpa-gu, Seoul 05505, Korea

Tel: $+82-2-3010-3263$

Fax: +82-2-3010-8047

E-mail: skpark@amc.seoul.kr
Background/Aims: Kidney transplantation (KT) reportedly provides a significant survival advantage over dialysis in diabetic patients. However, KT outcome in diabetic patients compared with that in non-diabetic patients remains controversial. In addition, owing to recent improvements in the outcomes of KT and management of cardiovascular diseases, it is necessary to analyze outcomes of recently performed KT in diabetic patients.

Methods: We reviewed all diabetic patients who received living donor KT between January 2008 and December 2011. Each patient was age- and sex-matched with two non-diabetic patients who received living donor KT during the same period. The outcomes of living donor KT were compared between diabetic and nondiabetic patients.

Results: Among 887 patients, 89 diabetic patients were compared with 178 nondiabetic patients. The incidence of acute rejection was not different between the diabetic and non-diabetic patients. Urinary tract infection and other infections as well as cardiovascular events occurred more frequently in diabetic patients. However, diabetes, cardiovascular disease, and infection were not significant risk factors of graft failure. Late rejection (acute rejection after 1 year of transplantation) was the most important risk factor for graft failure after adjusting for diabetes mellitus (DM), human leukocyte antigen mismatch, rejection and infection (hazard ratio, 56.082; 95\% confidence interval, 7.169 to 438.702 ; $p<0.001$ ). Mortality was not significantly different between diabetic and non-diabetic patients (o vs. $2, p=0.344$ by log-rank test).

Conclusions: End-stage renal disease patients with DM had favorable outcomes with living donor kidney transplantation.

Keywords: Kidney transplantation; Diabetes mellitus; Living donors

\section{INTRODUCTION}

The prevalence of diabetes mellitus (DM) has been increasing worldwide in recent decades, with the global prevalence estimated at $9 \%$ in 2014 , according to a re- port by the World Health Organization. The report also mentions that DM was directly responsible for 1.5 million deaths and 89 million disability-adjusted life years in 2012 [1]. The prevalence of DM has also been increasing in Korea [2]. Diabetic nephropathy is the most com- 
mon cause of end-stage renal disease (ESRD) in Korea, with an increase from 19.5\% in 1992 to $50.6 \%$ in 2012 [3].

Kidney transplantation reportedly provides a significant survival advantage over dialysis, and it is considered the best renal replacement option for diabetic ESRD patients $[4,5]$. In addition, several studies revealed that preemptive transplantation and living donor kidney transplantation were superior to deceased donor transplantation $[6,7]$. However, kidney transplantation outcome in diabetic patients compared with that in non-diabetic patients remains controversial. Some studies reported poor graft survival and mortality in diabetic patients [8-10], whereas others showed no significant differences in graft survival and mortality between diabetic and non-diabetic patients [11-14].

There are still patients and physicians who consider $\mathrm{DM}$ as deleterious to kidney transplantation outcomes. In addition, owing to recent improvements in the outcomes of kidney transplantation and management of cardiovascular diseases, it is necessary to analyze outcomes of recently performed kidney transplantation in diabetic patients. Furthermore, the type of DM is unclear in certain cases. Therefore, in this study, we compared the outcomes of recently performed kidney transplantation in diabetic patients with those in ageand sex-matched non-diabetic patients regardless of the etiology of ESRD and type of DM.

\section{METHODS}

\section{Study design}

In this retrospective study, we reviewed all patients who received kidney transplants between January 2008 and December 2011. Patients who were diagnosed with DM before transplantation and received living donor kidney transplants were included in this study. Patients who were under the age of 18 years and those who received other organ transplants were excluded. ABO incompatible or human leukocyte antigen (HLA) sensitized kidney transplantation were also excluded. In addition, only patients who were followed up for at least 1 year after transplantation were included. Each patient from the study group was age- and sex-matched with two non-diabetic patients who received living donor kidney transplants during the same period. The outcomes of living donor kidney transplantation were compared between diabetic and non-diabetic patients. The study was approved by Asan Medical Center Institutional Review Board (2016-0208).

\section{Transplantation protocol}

Patients received induction therapy with $20 \mathrm{mg}$ of basiliximab on the day of surgery and on postoperative day 4. Maintenance immunosuppressive regimen consisted of a calcineurin inhibitor (cyclosporine or tacrolimus), antimetabolites (mycophenolate mofetil or azathioprine), and a corticosteroid. Postoperatively, all patients received oral trimethoprim/sulfamethoxazole (80/400 $\mathrm{mg}$ ) for 6 months for pneumocystis pneumonia (PCP) prophylaxis. Cytomegalovirus (CMV) prophylactics were given routinely for CMV immunoglobulin G (IgG)-negative recipients matched with CMV IgG-positive donors. In other cases, preemptive therapy was administered based on serial monitoring for CMV antigenemia. All recipients underwent pre-transplant evaluation of cardiovascular risk by electrocardiogram, echocardiography and thallium-201 myocardial single photon emission computed tomography. When significant coronary artery disease was suspected, coronary angiogram was performed after discussion with cardiologists.

\section{Data collection and definition}

Baseline characteristics of patients, including age, sex, body mass index (BMI), past medical history, dialysis, and medications, were collected. Donor information and data on transplantation procedures was also reviewed. The occurrence of rejection, infection, cardiovascular events, and malignancy were also identified. Graft failure was defined as the recommencement of dialysis or direct second transplantation, and delayed graft function was defined as the use of dialysis in the first postoperative week [15]. Cardiovascular diseases included coronary artery disease, peripheral artery disease, and cerebral infarction. CMV infection was defined as $\geq 50$ CMV (+) cells/200,000 white blood cells using a CMV antigenemia assay and was treated with ganciclovir. When plasma BK virus (BKV) DNA levels were $>10,000$ copies/mL, BKV infection was diagnosed regardless of the presence of nephropathy. Protocol biopsies were not performed. Allograft biopsy was performed when rejection was clinically suspected. Renal biopsies were evalu- 
ated by light, electron, and immunofluorescence microscopy. C4d staining was performed on all specimens, and rejection was assessed with the Banff classification. The estimated glomerular filtration rate (eGFR) was calculated using the isotope-dilution mass spectrometrytraceable Modification of Diet in Renal Disease equation as follows: eGFR $\left(\mathrm{mL} / \mathrm{min} / 1.73 \mathrm{~m}^{2}\right)=175 \times$ serum creatinine $^{-1.154} \times$ age $^{-0.203} \times(0.742$ if patient is female $) \times$ (1.212 if patient is black).

\section{Statistical analysis}

Data were expressed as mean \pm standard deviation or number (percentage). For categorical variables, the chisquare test and Fisher exact test were used. Continuous variables were compared using the Student $t$ test or Mann-Whitney $U$ test. Graft survival and mortality rates were evaluated by the Kaplan-Meier method with logrank test. Cox proportional hazard analyses were used to assess variables associated with graft survival, using hazard ratios (HRs) and 95\% confidence intervals (CIs). Variables with a $p$ value of $<0.1$ in the univariate analysis were included in the initial step of multivariate analysis. All reported $p$ values were two-sided, and $p$ values of $<$ 0.05 were considered significant. All statistical analyses were performed using SPSS version 21.0 (IBM Corp., Armonk, NY, USA).

\section{RESULTS}

\section{Baseline characteristics}

A total of 887 patients received kidney transplantation between 2008 and 2011 at Asan Medical Center (Fig. 1), of which 129 patients were excluded and 112 diabetic patients were included. However, four diabetic patients could not be matched with non-diabetic patients within 5 years of difference in age. In addition, 19 patients who received ABO incompatible or HLA sensitized kidney transplantation were excluded. Therefore, in the final analysis, 89 diabetic patients were compared with 178 non-diabetic patients. The mean BMI of diabetic patients was higher than that of non-diabetic patients $\left(24.52 \pm 3.68 \mathrm{~kg} / \mathrm{m}^{2} \mathrm{vs}\right.$. $23.38 \pm 2.89 \mathrm{~kg} / \mathrm{m}^{2}, p=0.006$ ) (Table 1). Additionally, more diabetic patients than non-diabetic patients were diagnosed with cardiovascular disease before transplantation (21.3\% vs. 6.7\%, $p=0.001$ ). The number of patients who

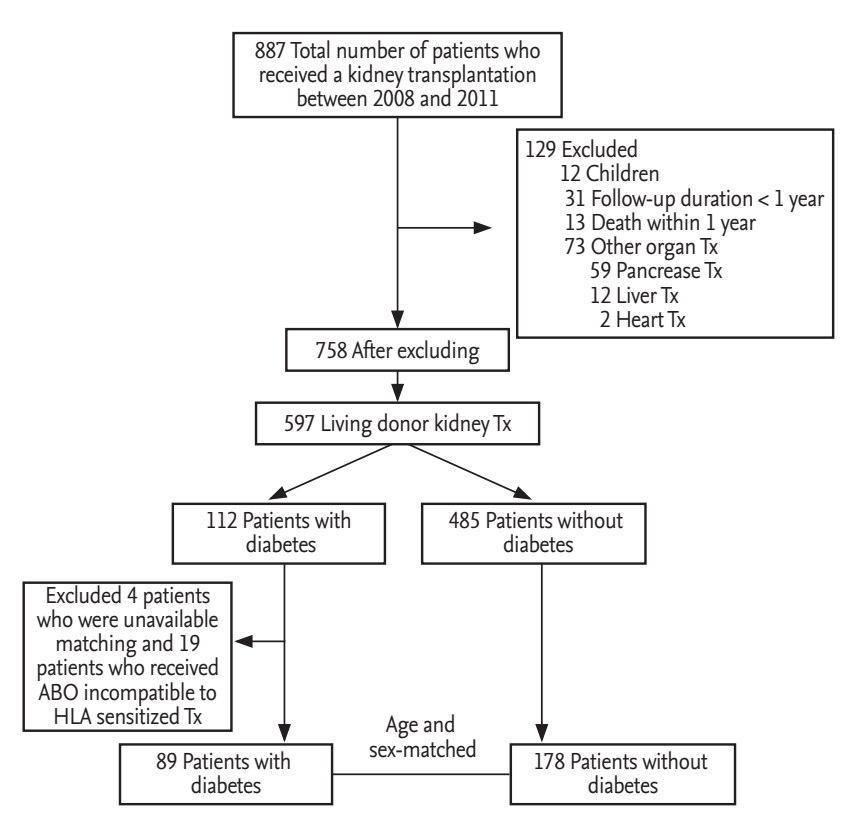

Figure 1. Flow chart of study population. Tx, transplantation; HLA, human leukocyte antigen.

were taking statin or renin-angiotensin blocker was not different between groups, but more patients were taking aspirin in diabetic patients (31.5\% vs. $9.6 \%, p<0.001$ ). Other baseline characteristics were not significantly different between the two groups.

\section{Acute rejection and infectious complications}

The incidence of acute rejection was not different between the diabetic and non-diabetic patients (Table 2). Delayed graft function occurred at similar rates in both groups (1.1\% vs. 1.1\%, $p=1$ ). The overall incidence of infectious complications within the first year after transplantation did not differ significantly between the two groups (Table 3). However, urinary tract infections and other infections occurred more frequently after the first year of transplantation in diabetic patients than in nondiabetic patients (12.4\% vs. $3.9 \%, p=0.017 ; 14.6 \%$ vs. $2.8 \%$, $p<0.001$, respectively). Finally, diabetic and non-diabetic patients showed similar incidence rates of CMV and BK virus infection.

\section{Graft survival and mortality}

The incidence of graft failure was $6.7 \%$ in diabetic patients and $2.8 \%$ in non-diabetic patients. In addition, graft survival rates at 1, 3, and 5 years were $100 \%, 100 \%$, and $95 \%$ in diabetic patients and $100 \%, 99 \%$, and $98 \%$ 
Baek $\mathrm{CH}$, et al. Living donor $\mathrm{KT}$ in diabetic patients

Table 1. Baseline characteristics of the study population

\begin{tabular}{|c|c|c|c|}
\hline Characteristic & DM patients $(\mathrm{n}=89)$ & Non-DM patients $(\mathrm{n}=178)$ & $p$ value \\
\hline Male sex, \% & 74.2 & 74.2 & 1.000 \\
\hline Age, yr & $50.36 \pm 8.63$ & $49 \cdot 38 \pm 7 \cdot 33$ & 0.334 \\
\hline Body mass index, $\mathrm{kg} / \mathrm{m}^{2}$ & $24.52 \pm 3.68$ & $23.38 \pm 2.89$ & 0.006 \\
\hline HbAic at 1 year after KT, \% & $8.0 \pm 1.37$ & & \\
\hline \multicolumn{4}{|l|}{ Medical history, \% } \\
\hline $\mathrm{DM}$ & 100 & 0 & $<0.001$ \\
\hline Hypertension & 92.1 & 90.4 & 0.821 \\
\hline Cardiovascular disease & 21.3 & 6.7 & 0.001 \\
\hline Hepatitis B & $7 \cdot 9$ & $7 \cdot 3$ & 1.000 \\
\hline Hepatitis C & 0 & 1.7 & 0.553 \\
\hline Dialysis prior transplantation, \% & 83.1 & 79.2 & 0.514 \\
\hline Dialysis modality, \% & & & 0.277 \\
\hline Hemodialysis & 85.1 & 78.0 & \\
\hline Peritoneal dialysis & $14 \cdot 9$ & 22.0 & \\
\hline Dialysis duration, mon & $20.26 \pm 21.35$ & $26.58 \pm 35 \cdot 55$ & 0.105 \\
\hline $\mathrm{KT} \geq 2$ times, $\%$ & $3 \cdot 4$ & $3 \cdot 9$ & 1.000 \\
\hline HLA mismatches $>3, \%$ & 41.6 & $34 \cdot 3$ & 0.282 \\
\hline $\mathrm{PRA} \geq 10 \%$ & 6.8 & 10.1 & 0.493 \\
\hline \multicolumn{4}{|l|}{ Immunosuppressant, \% } \\
\hline Tacrolimus & $57 \cdot 3$ & 48.9 & 0.242 \\
\hline Cyclosporine & 42.7 & 51.1 & 0.242 \\
\hline Mycophenolate & 78.7 & 73.6 & 0.452 \\
\hline Azathioprine & 11.2 & 14.0 & 0.570 \\
\hline Cyclophosphamide & $7 \cdot 9$ & 8.4 & 1.000 \\
\hline Everolimus & 2.2 & $3 \cdot 9$ & 0.722 \\
\hline \multicolumn{4}{|l|}{ Other medications, $\%$} \\
\hline Statins & 51.7 & 60.1 & 0.193 \\
\hline Renin-angiotensin blocker & 14.6 & 11.2 & 0.436 \\
\hline Aspirin & 31.5 & 9.6 & $<0.001$ \\
\hline \multicolumn{4}{|l|}{ Diabetes management, \% } \\
\hline Insulin & 51.7 & & \\
\hline Insulin + OHA & $39 \cdot 3$ & & \\
\hline $\mathrm{OHA}$ & 9.0 & & \\
\hline Donor sex (male), \% & 46.1 & 50.6 & 0.518 \\
\hline Donor age, yr & $41.17 \pm 12.29$ & $41.13 \pm 10.39$ & 0.979 \\
\hline Related donor, \% & $53 \cdot 9$ & 64.0 & 0.114 \\
\hline Follow-up duration, mon & $64.46 \pm 15.13$ & $66.77 \pm 15.03$ & 0.239 \\
\hline
\end{tabular}

Values are presented as mean \pm SD.

DM, diabetes mellitus; HbAıc, glycated hemoglobin; KT, kidney transplantation; HLA, human leukocyte antigen; PRA, panel reactive antibody; OHA, oral hypoglycemic agents. 
Table 2. Incidence of acute rejection

\begin{tabular}{lccc}
\hline Variable & DM patients $(\mathrm{n}=89)$ & Non-DM patients $(\mathrm{n}=178)$ & \\
\hline Within 1 year of KT, \% & & 8.4 & 0.655 \\
T cell mediated rejection & 10.1 & 0.6 & 1.000 \\
Antibody mediated rejection & 0 & 12.4 & 0.452 \\
After 1 year of KT, \% & & 5.6 & 0.780 \\
T cell mediated rejection & 15.7 & 4.5 & \\
Antibody-mediated rejection & & & \\
\hline
\end{tabular}

DM, diabetes mellitus; KT, kidney transplantation.

Table 3. Incidence of infection

\begin{tabular}{|c|c|c|c|}
\hline Variable & DM patients $(\mathrm{n}=89)$ & Non-DM patients $(n=178)$ & $p$ value \\
\hline Within 1 year of KT, \% & 32.6 & 30.3 & 0.779 \\
\hline Cytomegalovirus & 9.0 & 14.0 & 0.324 \\
\hline BK virus & 10.1 & 10.1 & 1.000 \\
\hline Pneumocystis & 1.1 & 0.6 & 1.000 \\
\hline Pneumonia & $3 \cdot 4$ & 2.2 & 0.689 \\
\hline Urinary tract infection & 12.4 & $7 \cdot 9$ & 0.267 \\
\hline Sepsis & 9.0 & 2.8 & 0.036 \\
\hline Others & 12.4 & 8.4 & 0.381 \\
\hline After 1 year of KT, \% & 23.6 & 12.9 & 0.035 \\
\hline Cytomegalovirus & 2.2 & 1.1 & 0.603 \\
\hline BK virus & 2.2 & 0.6 & 0.258 \\
\hline Pneumocystis & o & 1.1 & 0.554 \\
\hline Pneumonia & $4 \cdot 5$ & 5.1 & 1.000 \\
\hline Urinary tract infection & 12.4 & $3 \cdot 9$ & 0.017 \\
\hline Sepsis & $4 \cdot 5$ & $4 \cdot 5$ & 1.000 \\
\hline Others & 14.6 & 2.8 & 0.001 \\
\hline
\end{tabular}

DM, diabetes mellitus; KT, kidney transplantation.

in non-diabetic patients. The cause of graft failure was rejection in five of six graft failures in diabetic patients and four of five graft failures in non-diabetic patients. One patient in diabetic group experienced graft failure due to calcineurin inhibitor toxicity and rejection. The cause of graft failure was unknown in one patient in non-diabetic group. Both death-censored graft survival and non-death-censored graft survival were not significantly different between the two groups $(p=0.103$ and $p=0.257$ by log rank test) (Fig. 2). DM was not a significant risk factor for graft failure in univariate analysis (HR, 2.589; 95\% CI, 0.789 to 8.490; $p=0.117$ ) (Table 4). As summarized in Table 4, univariate analysis revealed that the risk of graft failure was significantly increased with HLA mismatches (4 to 6 mismatches) (HR, 4.794; 95\% CI, 1.272 to $18.074 ; p=0.021$, acute rejection within the first year after transplantation (HR, 4.889; 95\% CI, 1.280 to 18.666; $p=0.020)$ and acute rejection after the first year of transplantation (HR, 59.684; 95\% CI, 7.638 to 466.342 ; $p<0.001$ ). However, in multivariate analysis, a significantly higher risk of graft failure was observed with only acute rejection after the first year of transplantation (HR, 56.082; 95\% CI, 7.169 to 438.702; $p<0.001$ ) and high HLA mismatches (HR, 4.159; 95\% CI, 1.100 to $15.722 ; p=0.036$ ). Mortality was not significantly different between diabetic and non-diabetic patients (o vs. $2, p=0.344$ by log- 
rank test) (Fig. 3). One non-diabetic patient died due to progression of small cell lung cancer complicated with pneumonia 5 years and 9 months after transplantation, and the other died due to PCP complicated with candida sepsis 1 year and 2 months after transplantation.

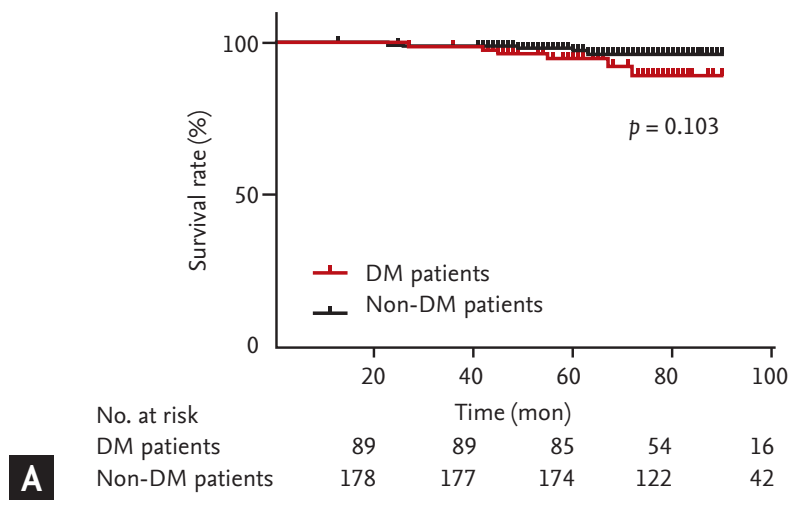

\section{The changes of graft function and proteinuria after transplantation}

Allograft function was not significantly different between diabetic patients and non-diabetic patients until 6 years after transplantation. More patients had proteinuria $\geq 2+$ on dipstick in diabetic group after 5 years of

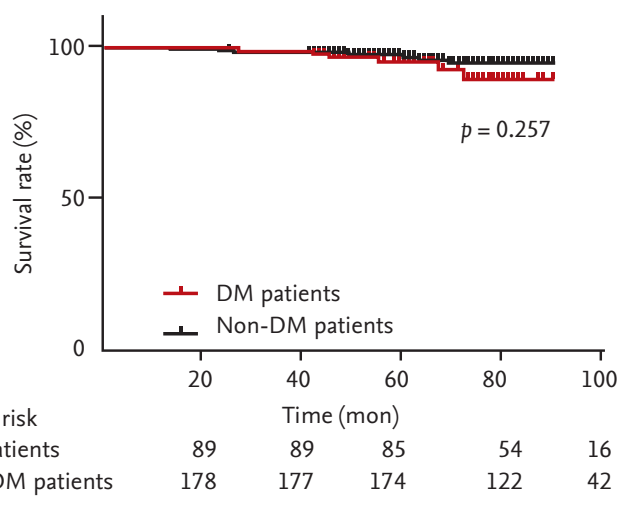

Figure 2. (A) Death-censored graft survival (B) non-death-censored graft survival. DM, diabetes mellitus.

Table 4. Unadjusted and adjusted risk factors for graft failure

\begin{tabular}{|c|c|c|c|c|}
\hline \multirow{2}{*}{ Variable } & \multicolumn{2}{|c|}{ Univariate analysis $^{\mathrm{a}}$} & \multicolumn{2}{|c|}{ Multivariate analysis ${ }^{\mathrm{b}}$} \\
\hline & $\operatorname{HR}(95 \% \mathrm{CI})$ & $p$ value & $\operatorname{HR}(95 \% \mathrm{CI})$ & $p$ value \\
\hline Diabetes mellitus & $2.589(0.789-8.490)$ & 0.117 & & \\
\hline Body mass index & $1.097(0.952-1.265)$ & 0.201 & & \\
\hline History of cardiovascular disease & $0.805(0.103-6.293)$ & 0.836 & & \\
\hline Dialysis modality & $1.108(0.230-5.336)$ & 0.898 & & \\
\hline Dialysis duration & $1.010(0.995-1.024)$ & 0.199 & & \\
\hline HLA mismatch & $4.794(1.272-18.074)$ & 0.021 & $4.159(1.100-15.722)$ & 0.036 \\
\hline Donor gender & $1.789(0.523-6.119)$ & 0.354 & & \\
\hline Donor age & $1.039(0.980-1.103)$ & 0.202 & & \\
\hline Unrelated donor & $2.948(0.863-10.077)$ & 0.085 & & \\
\hline Acute rejection within 1 year of KT & $4.889(1.280-18.666)$ & 0.020 & & \\
\hline Acute rejection after 1 year of KT & $59.684(7.638-466.342)$ & $<0.001$ & $56.082(7.169-438.702)$ & $<0.001$ \\
\hline Infectious complication within 1 year of KT & $1.307(0.381-4.483)$ & 0.671 & & \\
\hline Infectious complication after 1year of KT & $2.791(0.816-9.546)$ & 0.102 & & \\
\hline Cardiovascular complications & $3.979(0.509-31.106)$ & 0.188 & & \\
\hline
\end{tabular}

HR, hazard ratio; CI, confidence interval; HLA, human leukocyte antigen; KT, kidney transplantation.

${ }^{a}$ Univariate Cox proportional hazard analysis was used.

${ }^{b}$ Multivariate Cox proportional hazard analysis with a backward stepwise method was used. Variables with $p<0.10$ in the univariate analysis (HLA mismatch, acute rejection within 1 year of transplantation, acute rejection after 1 year of transplantation, unrelated donor) and diabetes mellitus were included in the initial step of multivariate analysis. Variables included in the final model are displayed. 
transplantation, but no significant difference was shown in other periods. Allograft function and proteinuria in each group are summarized in Table 5 .

\section{Cardiovascular events and malignancy}

More diabetic patients had cardiovascular disease before transplantation than non-diabetic patients. Likewise, diabetic patients experienced cardiovascular events more often than non-diabetic patients after transplantation (5.6\% vs. $0.6 \%, p=0.017$ ). Three of the five diabetic patients and one non-diabetic patient who experienced cardiovascular events after transplantation already had past histories of cardiovascular disease before transplantation. Two of the diabetic patients had coronary artery disease and three had both coronary artery disease and

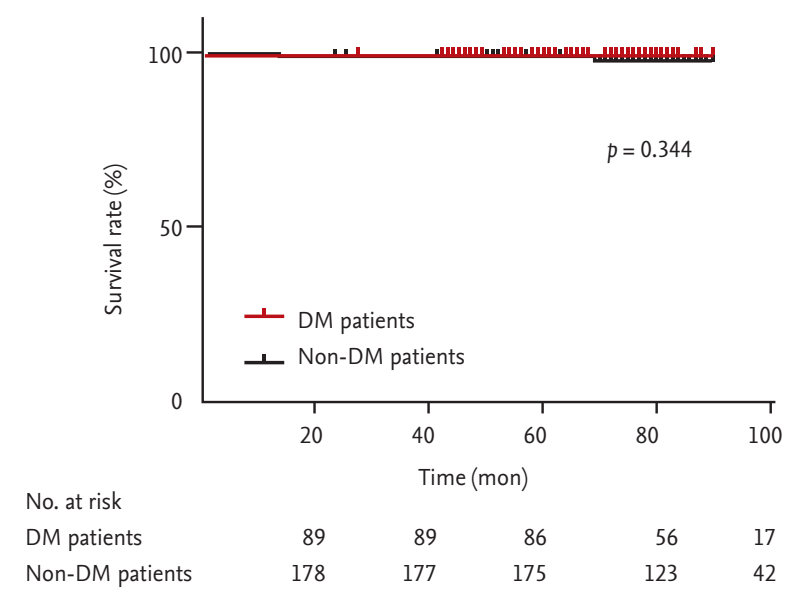

Figure 3. Mortality. DM, diabetes mellitus.

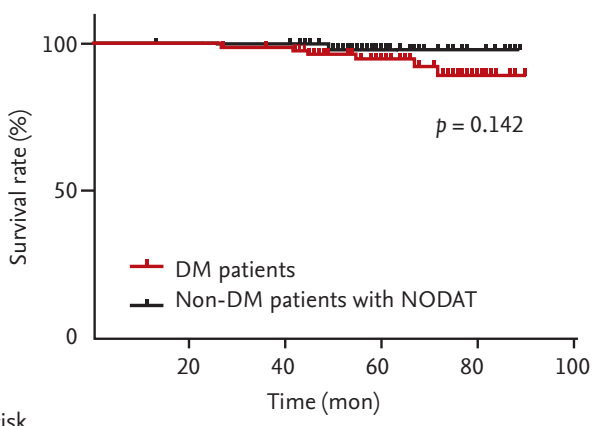

No. at risk

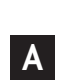
DM patients NODAT

$\begin{array}{lllll}89 & 89 & 85 & 54 & 16 \\ 62 & 61 & 61 & 38 & 14\end{array}$

peripheral artery disease. In contrast, a non-diabetic patient had only coronary artery disease. None of the patients in this study experienced stroke after transplantation. Finally, the incidence of malignancy of any type was not different between the two groups (4.5\% vs. $2.8 \%$, $p=0.487)$.

\section{Subgroup analysis of NODAT patients}

Among 178 non-diabetic patients, new-onset diabetes after transplantation (NODAT) developed in 62 patients (34.8\%) during follow-up. Graft survival of diabetic patients and non-diabetic patients with or without NODAT was not significantly different $(p=0.142$ and $p=$ 0.217 by log rank test) (Fig. 4). In the subgroup analysis of diabetic patients and non-diabetic patients without NODAT, the incidence of rejection and infection showed similar pattern with the results of overall analysis. The incidences of acute rejection within 1 year and after 1 year of transplantation were not different between the two groups $(p=0.623$ and $p=0.855)$. In addition, although total incidence of infection within 1 year did not show significant difference (32.6\% vs. $26.7 \%, p=0.439$ ), sepsis occurred in more patients in diabetic patients compared with non-diabetic patients without NODAT (9.0\% vs. $0.9 \%, p=0.011$ ). After 1 year of transplantation, the difference of overall incidence of infection between the two groups did not reach statistical significance $(23.6 \%$ vs. $13.8 \%, p=0.098)$. However, urinary tract infection and other infections occurred more often in diabetic patients after 1 year of transplantation ( $12.4 \%$ vs. $3.4 \%$, $p=0.027$ for urinary tract infection; $14.6 \%$ vs. $3.4 \%, p=$ 0.005). However, the incidence of CMV and BKV infection was not different between two groups.

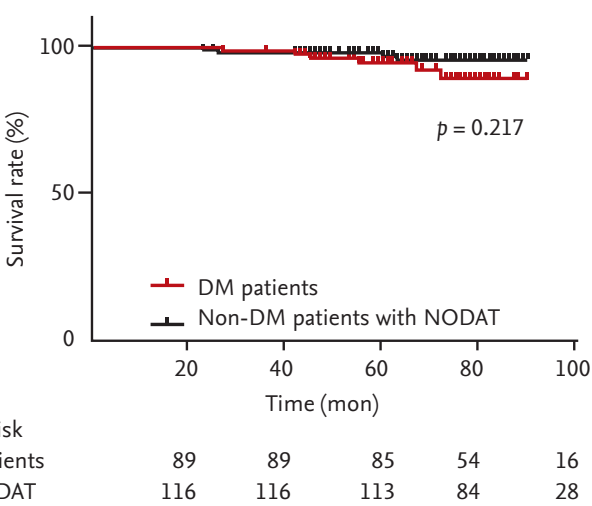

Figure 4. Graft survival of patients (A) with and (B) without new-onset diabetes after kidney transplantation. DM, diabetes mellitus; NODAT, new onset diabetes after transplantation. 
Table 5. Graft function and proteinuria after transplantation

\begin{tabular}{|c|c|c|c|}
\hline Variable & DM patients $(\mathrm{n}=89)$ & Non-DM patients $(\mathrm{n}=178)$ & $p$ value \\
\hline \multicolumn{4}{|l|}{ eGFR (no. of patients) } \\
\hline 1 Year after KT (89/178) & $62.40 \pm 13.20$ & $65.22 \pm 15.81$ & 0.148 \\
\hline 2 Years after KT (89/175) & $62.05 \pm 15.97$ & $65.01 \pm 14.62$ & 0.133 \\
\hline 3 Years after KT (87/173) & $65.40 \pm 20.40$ & $64.26 \pm 15.49$ & 0.616 \\
\hline 4 Years after KT (70/155) & $64.76 \pm 21.79$ & $64.78 \pm 16.58$ & 0.995 \\
\hline 5 Years after KT (51/119) & $64.49 \pm 18.68$ & $66.34 \pm 17.86$ & 0.541 \\
\hline 6 Years after KT (32/73) & $65.49 \pm 20.98$ & $66.98 \pm 15.54$ & 0.720 \\
\hline 7 Years after KT (8/24) & $73 \cdot 50 \pm 15 \cdot 54$ & $59 \cdot 54 \pm 14 \cdot 557$ & 0.028 \\
\hline \multicolumn{4}{|c|}{ Proteinuria $\geq 2+$ on dipstick,\% } \\
\hline 1 Year after KT (88/177) & 0 & 1.7 & 0.553 \\
\hline 2 Years after KT (89/174) & 1.1 & $\mathrm{O}$ & 0.338 \\
\hline 3 Years after KT (87/172) & $3 \cdot 4$ & 2.3 & 0.690 \\
\hline 4 Years after KT (70/154) & $4 \cdot 3$ & 1.3 & 0.178 \\
\hline 5 Years after KT (51/118) & 9.8 & 1.7 & 0.027 \\
\hline 6 Years after KT (32/73) & 6.3 & 0 & 0.091 \\
\hline 7 Years after KT (8/24) & 0 & 4.2 & 1.000 \\
\hline
\end{tabular}

Values are presented as mean $\pm \mathrm{SD}$.

DM, diabetes mellitus; eGFR, estimated glomerular filtration rate; KT, kidney transplantation

\section{DISCUSSION}

In the present study, kidney transplantation outcomes (graft survival, mortality, acute rejection, and delayed graft function) in diabetic patients were comparable with those in non-diabetic patients. Urinary tract infection and other infections as well as cardiovascular events occurred more frequently in diabetic patients. However, DM, cardiovascular disease, and infection were not significant risk factors of graft failure. Late rejection (acute rejection after 1 year of transplantation) was the most important risk factor for graft failure after adjusting for DM, HLA mismatch, rejection and unrelated donor.

Both death-censored graft survival and nondeath-censored graft survival were not different between the two patient groups. Although late rejection was the most important risk factor for graft failure, the incidence of acute rejection was not different between diabetic patients and non-diabetic patients. Regarding acute $\mathrm{T}$ cell mediated rejection, there was no significant difference in the Banff grade within 1 year of transplantation versus after 1 year of transplantation in this study. Further studies are necessary to elucidate whether diabetes increas- es rejection severity or refractoriness to the treatment of rejection. In addition, diabetic patients had higher prevalence of cardiovascular disease and other infections such as diabetic foot in this study. Therefore, other factors which were not evaluated in this study, such as more frequent exposure to radiocontrast agents or metabolic effects, might have contributed to renal function. Previous studies reported increased mortality related to cardiovascular disease in diabetic patients after kidney transplants [8]. However, in this study, no patient died from cardiovascular disease, and non-death-censored graft survival was comparable between the two groups. Improvements in pre-transplantation evaluation and management of cardiovascular disease might have contributed to these results. We have summarized the previous studies that have compared transplant outcomes between recipients with diabetes and without diabetes in Table $6[8-14,16,17]$.

In order to evaluate if the outcomes of kidney transplant recipients with diabetes have improved, we additionally analyzed recipients in 1995 to 1998 . There were 25 diabetic patients who received living donor kidney transplantation between 1995 and 1998. The 1-, 3- and 


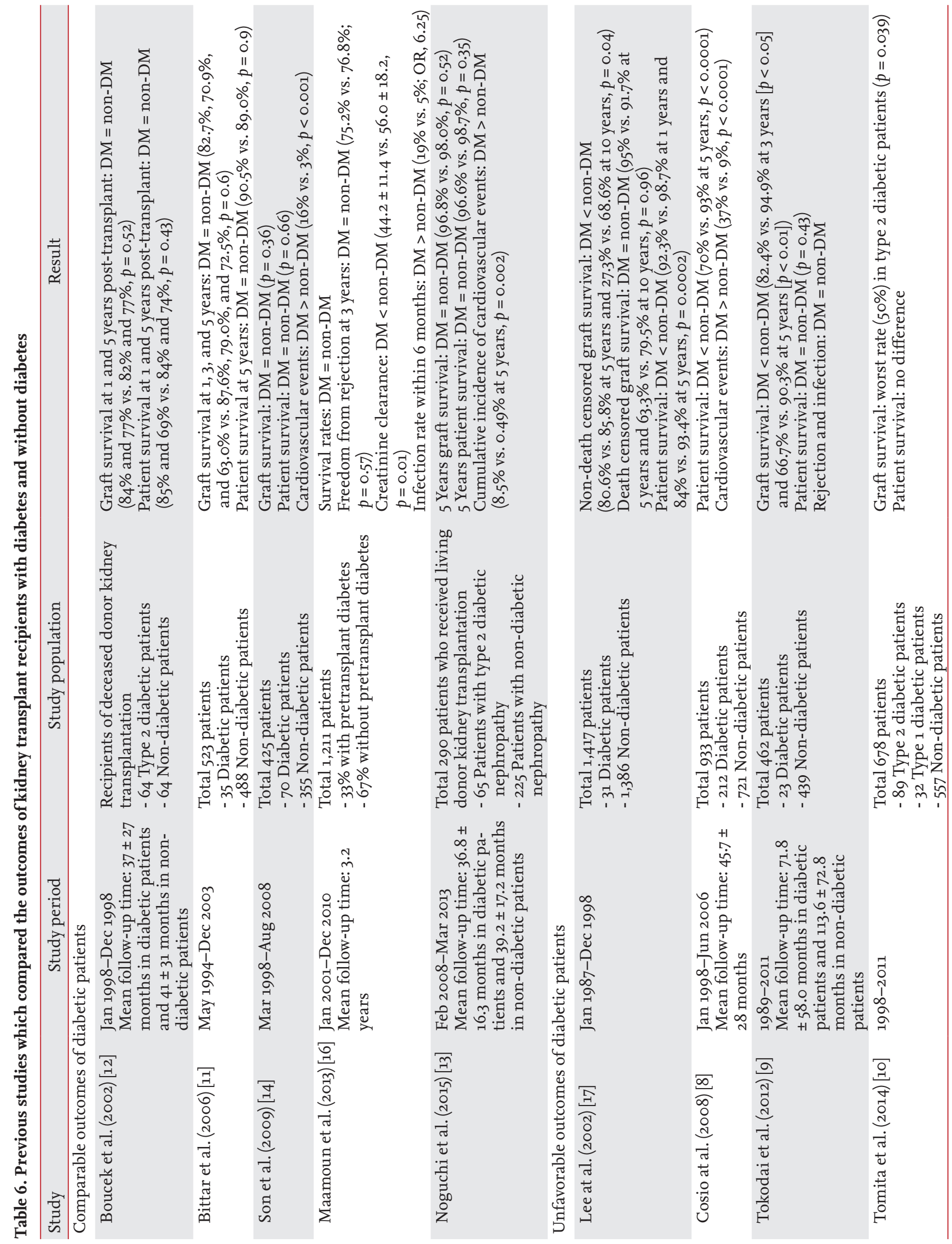


5-year graft survival were $96 \%, 88 \%$, and $88 \%$ in patients of 1995 to 1998 and $100 \%, 99 \%$, and $95 \%$ in patients of 2008 to 2011 . Three-year graft survival showed significant difference between the two groups $(p=0.033)$, but 5 -year graft survival was not different $(p=0.177)$. The mean follow-up duration were different between the two groups, and the number of patients was small. Therefore, longer follow-up of this study population is necessary.

Several studies suggested that hyperglycemia might be associated with an increased risk of acute allograft rejection in diabetic patients $[7,18]$. However, in the present study, the incidence of acute rejection was not higher in diabetic patients either in or after the first year of transplantation. Maamoun et al. [16] also reported that between diabetic and non-diabetic recipients, there were no significant differences in rejection rates at 1 year (16\% vs. $22 \%)$ or in freedom from rejection at 3 years $(75.2 \%$ vs. $76.8 \%, p=0.57)$. Strict glycemic control might be important for graft survival. Further studies to elucidate any relationship between DM and rejection are necessary in diabetic patients with transplants.

The incidence of infectious complications varies among studies. Lansang et al. [19] reported that the incidence of infections requiring hospitalizations, including septicemia, pneumonia, and urinary tract infection, was higher in diabetic patients with kidney transplants. In addition, Abbott et al. [20] reported that DM in transplant recipients was the only independent risk factor for fungal infections. However, several other reports showed that the infection rate was not different between diabetic patients and non-diabetic patients $[9,13]$. In a study by Tokodai et al. [9], there were no significant differences in total infection or urinary tract infection rates. In our study, the overall incidence of infection was not different between groups. However, sepsis (within 1 year of transplantation) and urinary tract infection and other infections (after 1 year of transplantation) occurred more frequently in diabetic patients, albeit with no significant impact on graft survival.

Several studies reported that transplant recipients with DM had a higher incidence of cardiovascular events $[7,8,13,21]$. Cosio et al. [8] compared the cardiovascular risk between diabetic and non-diabetic recipients and showed that diabetic patients had reduced 5-year survival rate $(70 \%$ vs. $93 \%, p<0.001)$ and higher incidence of cardiovascular events (37\% vs. $9 \%, p<0.001$ ). In non-diabetic patients, the incidence of post-transplant cardiovascular events was related to traditional cardiovascular risk factors; only the pre-transplant cardiovascular history was related to this outcome in diabetic patients. In the present study, more diabetic patients had a history of cardiovascular disease, and cardiovascular events occurred more frequently in diabetic patients after transplantation. However, the causes of death were malignancy progression and uncontrolled infections and no patient died from cardiovascular events. Long-term follow-up of these patients will allow for more definite results. This study has several limitations. First, this is a retrospective study based on patient medical records, so not all information could be obtained. Second, the follow-up period of patients was relatively short (mean, approximately 5 years); longer follow-up periods will allow for accumulation of more information on the outcomes of kidney transplantation in diabetic patients.

In conclusion, ESRD patients with DM had favorable outcomes with living donor kidney transplantation. The presence of DM, infection, and cardiovascular disease were not significant contributors of graft failure in patients with recently performed transplantation. Therefore, clinicians should consider recommending kidney transplantation to ESRD patients with diabetes. The incidence of cardiovascular events was still high in diabetic patients; thus, close preoperative evaluation and postoperative monitoring are necessary.

\section{KEY MESSAGE}

1. End-stage renal disease (ESRD) patients with diabetes mellitus (DM) had favorable outcomes with recently performed living donor kidney transplantation.

2. Acute rejection was the most important risk factor for graft failure after adjusting for DM, human leukocyte antigen mismatch, rejection, and types of donors.

3. Clinicians should consider recommending kidney transplantation to ESRD patients with diabetes. 


\section{Conflict of interest}

No potential conflict of interest relevant to this article was reported.

\section{REFERENCES}

1. World Health Organization (WHO). Global Status Report on Noncommunicable Diseases 2014. Geneva: WHO, 2014.

2. Ha KH, Kim DJ. Trends in the diabetes epidemic in Korea. Endocrinol Metab (Seoul) 2015;30:142-146.

3. Jin DC, Han JS. Renal replacement therapy in Korea, 2012. Kidney Res Clin Pract 2014;33:9-18.

4. Luan FL, Samaniego M. Transplantation in diabetic kidney failure patients: modalities, outcomes, and clinical management. Semin Dial 2010;23:198-205.

5. Wolfe RA, Ashby VB, Milford EL, et al. Comparison of mortality in all patients on dialysis, patients on dialysis awaiting transplantation, and recipients of a first cadaveric transplant. N Engl J Med 1999;341:1725-1730.

6. Becker BN, Rush SH, Dykstra DM, Becker YT, Port FK. Preemptive transplantation for patients with diabetes-related kidney disease. Arch Intern Med 2006;166:44-48.

7. Guerra G, Ilahe A, Ciancio G. Diabetes and kidney transplantation: past, present, and future. Curr Diab Rep 2012;12:597-603.

8. Cosio FG, Hickson LJ, Griffin MD, Stegall MD, Kudva Y. Patient survival and cardiovascular risk after kidney transplantation: the challenge of diabetes. Am J Transplant 2008;8:593-599.

9. Tokodai K, Amada N, Kikuchi H, Haga I, Takayama T, Nakamura A. Outcomes of renal transplantation after end-stage renal disease due to diabetic nephropathy: a single-center experience. Transplant Proc 2012;44:77-79.

10. Tomita Y, Iwadoh K, Kutsunai K, Koyama I, Nakajima I, Fuchinoue S. Negative impact of underlying non-insulin-dependent diabetes mellitus nephropathy on longterm allograft survival in kidney transplantation: a 10year analysis from a single center. Transplant Proc
2014;46:3438-3442.

11. Bittar J, Cepeda P, de la Fuente J, Douthat W, de Arteaga J, Massari PU. Renal transplantation in diabetic patients. Transplant Proc 2006;38:895-898.

12. Boucek P, Saudek F, Pokorna E, et al. Kidney transplantation in type 2 diabetic patients: a comparison with matched non-diabetic subjects. Nephrol Dial Transplant 2002;17:1678-1683.

13. Noguchi H, Kitada H, Kaku K, et al. Outcome of renal transplantation in patients with type 2 diabetic nephropathy: a single-center experience. Transplant Proc 2015;47:608-611.

14. Son YK, Oh JS, Oh HJ, Shin YH, Kim JK. Clinical outcome of kidney transplantation in patients with diabetic nephropathy. Korean J Med 2009;77:321-327.

15. Mallon DH, Summers DM, Bradley JA, Pettigrew GJ. Defining delayed graft function after renal transplantation: simplest is best. Transplantation 2013;96:885-889.

16. Maamoun HA, Soliman AR, Fathy A, Elkhatib M, Shaheen N. Diabetes mellitus as predictor of patient and graft survival after kidney transplantation. Transplant Proc 2013;45:3245-3248.

17. Lee SC, Youn HJ, Goo YS, et al. Kidney transplantation in diabetic patients: clinical study in a single center. J Korean Soc Transplant 2002;16:183-188.

18. Thomas MC, Mathew TH, Russ GR, Rao MM, Moran J. Early peri-operative glycaemic control and allograft rejection in patients with diabetes mellitus: a pilot study. Transplantation 2001;72:1321-1324.

19. Lansang MC, Ma L, Schold JD, Meier-Kriesche HU, Kaplan B. The relationship between diabetes and infectious hospitalizations in renal transplant recipients. Diabetes Care 2006;29:1659-1660.

20. Abbott KC, Hypolite I, Poropatich RK, et al. Hospitalizations for fungal infections after renal transplantation in the United States. Transpl Infect Dis 2001;3:203-211.

21. Kiberd B, Panek R. Cardiovascular outcomes in the outpatient kidney transplant clinic: the Framingham risk score revisited. Clin J Am Soc Nephrol 2008;3:822-828. 